\title{
AVALIAÇÃO DA SAÚDE E QUALIDADE DE VIDA: PROFISSIONAIS DE UM SAMU*
}

\author{
Eniva Miladi Fernandes Stumm¹, Geísa Ribeiro², Rosane Maria Kirchner ${ }^{3}$, Marli Maria Loro4, Cleci Lourdes Schmidt
} Piovesan Rosanelli ${ }^{5}$

\begin{abstract}
RESUMO: Profissionais que atuam em Atendimento Pré-Hospitalar têm como cenário um ambiente tenso, em muitos casos, com risco de comprometimento da saúde, exigindo conhecimento técnico-científico, segurança e estabilidade emocional. A pesquisa analisa o perfil de uma equipe de profissionais que atua em um Serviço de Atendimento PréHospitalar, buscando relacioná-lo à saúde e à qualidade de vida. É quantitativa, descritiva, transversal, realizada no Serviço de Atendimento Móvel de Urgência-SAMU, de Florianópolis-Santa Catarina, em outubro de 2008. O perfil dos trabalhadores pesquisados é: homens e mulheres, em percentuais aproximados, jovens, casados, com filhos, especialistas, enfermeiros e médicos, atuando de 5 a 10 anos na profissão. A maioria optou por atuar no SAMU, mais da metade, sem exclusividade. Eles sentem-se satisfeitos com sua saúde e a maioria, igualmente, avalia sua qualidade de vida de forma positiva, independente do gênero e da idade.
\end{abstract}

PALAVRAS-CHAVE: Assistência pré-hospitalar; Profissional de saúde; Saúde; Qualidade de vida.

\section{HEALTH AND LIFE QUALITY EVALUATION ON PROFESSIONALS OF ASAMU}

ABSTRACT: Professionals in pre hospital attendance-APH have as work environment a tense scenery, in most cases, involving health compromising situations, requiring scientific technical knowledge, security and emotional stability. The research analyzes the profile of health professionals team at the pre hospital attendance, trying to figure out a relationship with health and life quality. It is a quantitative, descriptive, and transverse study, developed on the Mobile Emergency Attendance Service-SAMU, in Florianópolis/ Santa Catarina, in October, 2008.The profile of the health workers are: women and men, in approximate percentages, young, married, with children, specialists, nurses and doctors, acting for 5 to 10 years on the health area. More than half of them chose to act on the SAMU, without exclusivity. They feel satisfied with their health and most of them evaluate their life quality in a positive manner, regardless of sex or gender.

KEYWORDS: Prehospital care; Health personnel; Health; Quality of life.

\section{EVALUACIÓN DE LA SALUD Y CALIDAD DE VIDA: PROFESIONALES DE UN SAMU}

RESUMEN: Profesionales que actúan en Atención Pre-Hospitalaria-APH tienen como escenario un ambiente tensionado, en muchos casos, con riesgo de comprometimiento de la salud, exigiendo conocimiento técnico-científico, seguridad y estabilidad emocional. La investigación analiza el perfil de un equipo de profesionales que actúa en un servicio de Atención Pre-Hospitalaria-APH, buscando relacionarlo a la salud y a la calidad de vida. Es cuantitativa, descriptiva, transversal, realizada en el Servicio de Atención Móvil de Urgencia - SAMU, de Florianópolis-Santa Catarina, en octubre de 2008. El perfil de los trabajadores investigados son hombres y mujeres, en porcentuales aproximados, jóvenes, casados, con hijos, expertos, enfermeros y médicos, actuando de 5 a 10 años en esta profesión. La mayoría optó por actuar en el SAMU, más de la mitad, sin exclusividad. Ellos se sienten satisfechos con su salud y, la mayoría, igualmente, evalúa su calidad de vida de forma positiva, independiente del género y de la edad.

PALABRAS CLAVE: Atención pre-hospitalaria; Profesional de salud; Salud; Calidad de vida.

\footnotetext{
*Artigo produzido de resultados de um Trabalho de Conclusão de Curso de Graduação de Enfermagem, defendido em dezembro de 2008, na Universidade Regional do Noroeste do Estado do Rio Grande do Sul-Unijui-RS.

${ }^{1}$ Enfermeira. Mestre em Administração-RH pela Universidade Federal do Rio Grande do Sul-UFRGS. Docente do Departamento de Ciências da Saúde da Universidade Regional do Noroeste do Estado do Rio Grande do Sul-Unijuí-RS.

${ }^{2}$ Enfermeira. Graduada em Enfermagem pela Unijui-RS.

${ }^{3}$ Doutora em Engenharia Elétrica. Métodos de Apoio à Decisão. Professora de Estatística do Campus da Universidade Federal do Pampa-UNIPAMPA.

${ }^{4}$ Enfermeira. Mestre em Educação nas Ciências. Docente do Departamento de Ciências da Saúde da Unijuí-RS.

${ }^{5}$ Enfermeira. Mestre em Educação nas Ciências. Docente do Departamento de Ciências da Saúde da Unijuí-RS.
}

Autor correspondente:

Eniva Miladi Fernandes Stumm

Universidade Federal do Rio Grande do Sul

Rua 20 de Setembro, 902 - 98700-000 - Ijui-RS

Recebido: 07/05/09

E-mail:eniva@unijui.edu.br

Aprovado: 02/10/09

Cogitare Enferm 2009 Out/Dez; 14(4):620-7 


\section{INTRODUÇÃO}

Nas unidades que prestam atendimento préhospitalar são realizados inúmeros procedimentos, desde os mais simples até os que envolvem alta complexidade. São locais onde os profissionais se deparam, cotidianamente, com situações inesperadas, que envolvem sofrimento, dor, morte e, muitas vezes, colocam em risco suas próprias vidas.

Eles vivenciam situações em que os familiares das vítimas igualmente necessitam de cuidados. Daí a necessidade de um atendimento rápido, eficiente e eficaz às vitimas no local do evento, sendo iniciada e prestada a intervenção assistencial pré-hospitalar, articulada com os demais serviços, especialmente o hospitalar, para que seja garantido o atendimento integral ao indivíduo, compatível com suas necessidades. Neste sentido,

prestar um cuidado adequado a essas vítimas pressupõe a existência de um sistema de atendimento de urgência que inclua um serviço de atenção pré-hospitalar articulado a hospitais com crescentes níveis de complexidade $\mathrm{e}^{(1: 287)}$.

Atendimento pré-hospitalar-APH é caracterizado como toda assistência prestada fora do ambiente hospitalar, de forma direta ou indireta, com o intuito de oferecer uma resposta apropriada. Esta varia desde um simples conselho até o envio de uma viatura de suporte básico ou avançado ao local da ocorrência, conforme a solicitação do usuário.

Os agravos que os usuários sofrem podem ser de natureza traumática, psiquiátrica, clínica, cirúrgica ou obstétrica, exigindo dos profissionais habilidades para prestar atendimento imediato, dispensando o mínimo de tempo nas ações de intervenção e de resgate, contribuindo para aumentar o nível de sobrevida, evitar e minimizar sequelas. Este atendimento pode ser de suporte básico ou avançado de vida. O suporte básico à vida não realiza manobras invasivas, porém o suporte avançado possibilita procedimentos invasivos de suporte ventilatório e circulatório. O cuidado qualificado no local do acidente, o encaminhamento e a chegada rápida ao hospital são decisivos para que a vítima sobreviva ${ }^{(2)}$.

O estado físico e psíquico dos profissionais que atuam em $\mathrm{APH}$, quando alterados, repercute no desempenho profissional, bem como na vida pessoal e familiar. A qualidade de vida-QV dos respectivos profissionais está diretamente relacionada à realização profissional, depende da valorização, do desenvolvimento e do reconhecimento que lhes é oferecido por meio de uma relação humanística ${ }^{(3)}$. Importante destacar que a QV está relacionada às percepções que o ser humano tem sobre os aspectos físicos, psicológicos, sociais, culturais, elementos importantes para sua condição humana, tanto no âmbito individual quanto coletivo. Ela é uma expressão de difícil conceituação, devido ao caráter subjetivo, complexo e multidimensional ${ }^{(4)}$. Ter QV depende de fatores intrínsecos e extrínsecos. Desta forma, existe uma conotação diferente de qualidade de vida para cada pessoa, resultante da sua inserção na sociedade.

Compete aos profissionais que atuam nos serviços de APH realizar um trabalho integrado, com destreza, agilidade, fundamentação teórica, preparo físico e estabilidade emocional. Viver neste ambiente pode contribuir para a tensão e o estresse, tendo como cenário situações que envolvem sofrimento, dor, angústia, medo, tensão e morte. Estas podem influenciar tanto na percepção acerca das situações avaliadas pelos sujeitos como estressantes, quanto nas respostas deles ao estresse, possivelmente repercutindo no desempenho profissional, na vida pessoal, familiar e social e, como consequência, interferir negativamente na qualidade de vida.

Assim, busca-se com a presente pesquisa analisar o perfil de uma equipe de profissionais que atua em um Serviço de Atendimento Pré-Hospitalar, buscando relacioná-lo às suas percepções referentes à avaliação da saúde e à qualidade de vida.

\section{MÉTODO}

O estudo se caracteriza como uma pesquisa quantitativa, descritiva, transversal, realizada no Serviço de Atendimento Móvel de Urgência-SAMU, na cidade de Florianópolis, Santa Catarina, em outubro de 2008. Dos 80 profissionais que atuam no SAMU, 41 aceitaram participar da pesquisa, incluindo 12 médicos, 14 enfermeiros, 7 técnicos em enfermagem, 4 socorristas e 4 motoristas socorristas.

O SAMU foi criado pela Secretária de Estado de Saúde de Santa Catarina, juntamente com o Ministério da Saúde e as Secretarias de Saúde do Estado. Funciona 24 horas, oferece à população assistência de profissionais de saúde compostas por médicos, enfermeiros, auxiliares de enfermagem e socorristas, totalizando 80 profissionais. A solicitação de assistência ao referido serviço ocorre por contato 
telefônico, atendido por técnicos da Central de Regulação, identificando a emergência e transferindo a ligação ao médico regulador, que realiza o diagnóstico e inicia o atendimento, passando as primeiras orientações ao usuário ou ao familiar.

O SAMU dispõe de Unidades de Suporte Básico de Vida, Unidade de Tratamento Intensivo Móvel e Helicóptero de Suporte Avançado de Vida, em parceria com o Corpo de Bombeiros, Militares e Voluntários, Policia Militar, Departamento de Policia Rodoviária Federal e Departamento Estadual de Defesa Civil.

Quanto ao plano de coleta de dados, utilizou-se um instrumento contendo dados de identificação e sociodemográficos, acrescido de duas questões, uma referente à satisfação com a saúde e outra à qualidade de vida. As referidas questões integram o instrumento versão em português abreviada da escala para avaliação da Qualidade Vida - WHOQOL - bref da Organização Mundial da Saúde-OMS. Para a análise dos dados utilizou-se o software estatístico SPSS e estatística descritiva. Os dados são apresentados em gráficos, tabelas simples e cruzadas.

Todos os aspectos éticos de uma pesquisa que envolve pessoas foram observados ${ }^{(5)}$. O projeto de pesquisa foi aprovado pelo Comitê de Ética em Pesquisa do Estado de Santa Catarina, Secretaria da Saúde, Diretoria de Educação Permanente em Saúde, sob Parecer Consubstanciado n. 0021.0809-08.

\section{RESULTADOS E DISCUSSÃO DOS DADOS}

Na Tabela 1, são apresentados os dados de identificação e socio-demográficas dos 41 profissionais que participaram desta pesquisa.

Quanto ao gênero dos profissionais (Tabela1), constata-se que o percentual de mulheres é semelhante ao de homens. Considerando o percentual de mulheres e buscando relacioná-lo ao estresse e à qualidade de vida, as mulheres, embora mais estressadas do que os homens, sabem lidar melhor com o estresse do que eles e apresentam menos doenças dele decorrentes, pelo fato de verbalizarem seus sentimentos e problemas no dia-a-dia ${ }^{(6)}$. Segundo a autora, para a sociedade a mulher deve ter uma postura adequada, trabalhar fora e, ao mesmo tempo, não abdicar de suas responsabilidades domésticas, como cuidar dos filhos. Nesse contexto, gradativamente, a mulher está conquistando seu espaço, buscando equilíbrio entre sucesso profissional e vida pessoal, bem como sua independência financeira.
Tabela 1 - Características sociodemográficas dos profissionais que atuam no SAMU de Florianópolis, Santa Catarina. Out-2008

\begin{tabular}{|c|c|c|}
\hline Característica & N. ${ }^{\circ}$ & $\%$ \\
\hline \multicolumn{3}{|l|}{ Gênero } \\
\hline Feminino & 20 & 48,8 \\
\hline Masculino & 21 & 51,2 \\
\hline \multicolumn{3}{|l|}{ Idade } \\
\hline $25 \mid---30$ anos & 11 & 26,8 \\
\hline 30|--- 35 anos & 14 & 34,1 \\
\hline $35 \mid---40$ anos & 8 & 19,5 \\
\hline 40|--- 45 anos & 6 & 14,6 \\
\hline 45 anos ou mais & 2 & 4,9 \\
\hline \multicolumn{3}{|l|}{ Estado Civil } \\
\hline Casado & 24 & 58,5 \\
\hline Solteiro & 15 & 36,6 \\
\hline Divorciado & 2 & 4,9 \\
\hline \multicolumn{3}{|l|}{ Escolaridade } \\
\hline Ensino Médio & 11 & 26,8 \\
\hline Graduação & 11 & 26,8 \\
\hline Especialização & 18 & 43,9 \\
\hline Mestrado & 1 & 2,4 \\
\hline \multicolumn{3}{|l|}{ Categoria Profissional } \\
\hline Enfermeiro(a) & 14 & 34,1 \\
\hline Médico(a) & 12 & 29,3 \\
\hline Técnico(a) em Enfermagem & 7 & 17,1 \\
\hline Motorista Socorrista & 4 & 9,8 \\
\hline Socorrista & 4 & 9,8 \\
\hline \multicolumn{3}{|l|}{ Filhos } \\
\hline Sim & 25 & 61,0 \\
\hline Não & 16 & 39,0 \\
\hline
\end{tabular}

Analisando a idade dos profissionais (Tabela1), evidencia-se que se trata de uma população jovem, em plena fase produtiva, sendo que o maior percentual situa-se na faixa etária de 30-35 anos incompletos, seguido dos de 25-30 anos incompletos. $\mathrm{O}$ fato de ser uma população predominantemente jovem merece atenção do gestor no sentido de que se associada ao estresse e à QV, trabalhadores jovens necessitam aprender a lidar com as demandas do ambiente de trabalho e podem apresentar maiores níveis de estresse, pela necessidade de desenvolver autonomia 
e responsabilidade. Associando este resultado à Síndrome de Burnout, quanto mais jovens os profissionais, maior é a Exaustão Emocional ${ }^{(7)}$.

Uma pesquisa com 17 enfermeiros de centro cirúrgico e recuperação anestésica, em Santa MariaRS, visando conhecer estressores e estratégias de coping, mostrou que quanto maior a idade do enfermeiro, menor é o valor total do coping dos fatores de confronto, suporte social, aceitação de responsabilidades e reavaliação positiva, ou seja, menos o enfermeiro os utiliza ${ }^{(8)}$.

Quanto ao estado civil dos profissionais, um percentual próximo a $60 \%$ é de casados e, ao associar este resultado a variável filho, constata-se que um percentual aproximado os possui (Tabela1). Mais de $40 \%$ deles são especialistas e, em torno de $25 \%$ graduados. Este resultado comprova que as pessoas estão investindo em sua formação profissional, buscando atualização na área em que atuam. Já, na variável categoria profissional, constata-se que os maiores percentuais são de enfermeiros e de médicos, seguido de técnicos em enfermagem. Este resultado é condizente com o tipo de unidade em que a pesquisa foi realizada. Importante ressaltar que ambas as categorias profissionais são consideradas por vários autores como estressantes.

Os percentuais de profissionais com especialização e graduação mostram que eles são qualificados para atuarem no referido serviço e esse resultado pode influenciar positivamente, tanto nas percepções deles quanto no enfrentamento de situações vivenciadas no ambiente de trabalho. Podese supor que profissionais com escolaridade mais elevada tenham mais expectativas em relação ao trabalho ${ }^{(9)}$. Nesse contexto, a qualificação dos profissionais tem sido primordial para o sucesso e desenvolvimento dos trabalhadores e das organizações em que atuam ${ }^{(10)}$. Em se tratando, especificamente, de APH, pesquisa com 23 profissionais do SAMU de Florianópolis mostrou que a respectiva área exige uma gama de conhecimentos, atuação específica e formação da equipe compatível com as demandas da população $0^{(11)}$.

Quanto ao tempo de atuação profissional, bem como de atuação no SAMU, constata-se que um percentual próximo a $60 \%$ possui de 5 a 10 anos incompletos de profissão e que mais de $25 \%$, de 10 a 20 anos. Esses resultados confirmam que se trata de uma população em plena fase produtiva e, ao associar esses resultados ao tempo em que eles atuam no
SAMU, a maioria está há menos de 5 anos. O tempo de atuação desses profissionais é um indicador importante de gestão e o fato de um elevado percentual deles atuar em outros locais merece atenção, no sentido de prevenir a Síndrome de Burnout, que emerge como resultado do estresse crônico, muitas vezes, inviabilizando o trabalhador de continuar exercendo suas atividades profissionais.

Nesse contexto, pesquisa com 224 empregados de uma empresa de auto-gestão de planos de previdência privada e de saúde de São Paulo-SP, mostrou que trabalhadores com menos tempo de atuação apresentaram nível maior de satisfação em relação ao trabalho ${ }^{(12)}$. Importante ressaltar que "um dos fatores de desgaste físico e psicológico para os trabalhadores da área da saúde é o acúmulo de dois ou mais vínculos empregatícios”(13:306).

Analisando as respostas dos profissionais pesquisados referentes à satisfação com a saúde (Figura1) evidencia-se que a maioria se sente satisfeito ou muito satisfeito. Chama atenção o fato de mais de $10 \%$ deles se sentirem insatisfeitos ou muito insatisfeitos e um percentual aproximado deste, se manter neutro, ou seja, nem satisfeito nem insatisfeito.

Resultado semelhante foi encontrado em pesquisa realizada em um hospital universitário, para analisar a qualidade de vida de 89 enfermeiros, 14 fisioterapeutas e 6 nutricionistas. Os autores constataram que a satisfação com a saúde somou 52 respostas para satisfeitos e 16 para muito satisfeito.Vale destacar que 14 enfermeiros relataram insatisfação com sua saúde ${ }^{(14)}$.

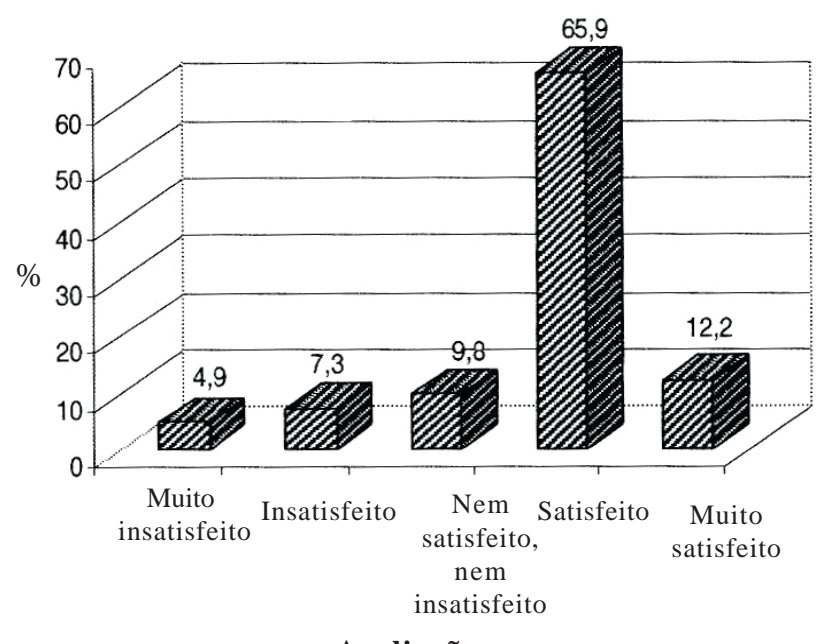

Avaliação

Figura1 - Frequência com que os profissionais do SAMU avaliam a satisfação com sua saúde. Florianópolis, Santa Catarina. Out-2008 


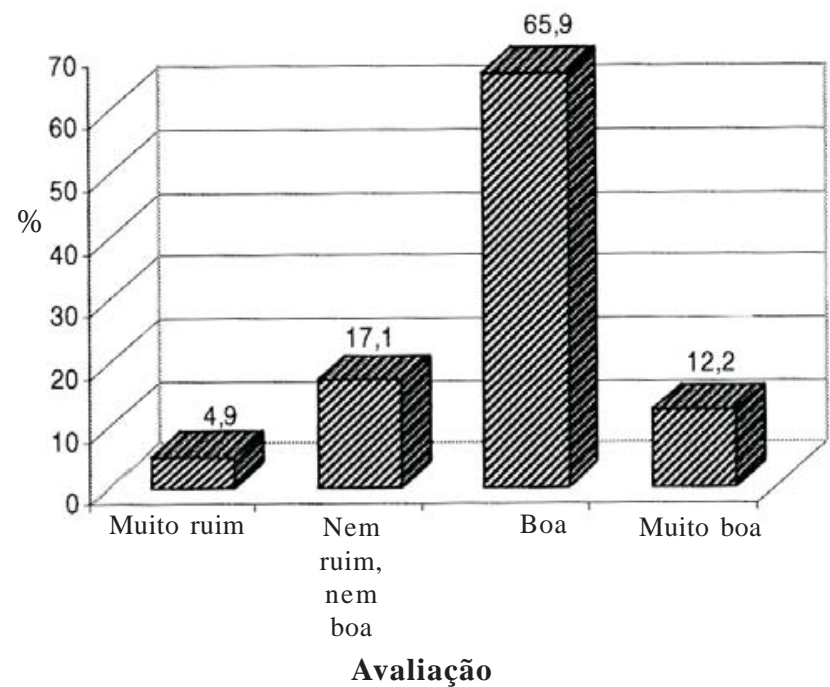

Figura 2 - Frequência com que os profissionais do SAMU avaliam sua Qualidade de Vida. Florianópolis, Santa Catarina. Out-2008

Na Figura 2, são explicitadas as respostas dos profissionais sobre como avaliam a sua qualidade de vida. Constata-se que a maioria avalia como boa e muito boa, o que é positivo, tanto para os trabalhadores quanto para a organização. Por outro lado, não se pode desconsiderar que um percentual próximo a $20 \%$ dos profissionais que participaram dessa pesquisa avalia sua qualidade de vida como nem boa nem ruim e os demais como muito ruim. Esse resultado é merecedor de estudos posteriores, buscando identificar com maior precisão os fatores que podem estar contribuindo para que esses trabalhadores avaliem a sua QV desta forma, bem como para reduzi-los.

Na mesma pesquisa, citada anteriormente, realizada em um hospital universitário, para analisar a qualidade de vida de 109 profissionais da saúde (enfermeiros, fisioterapeutas e nutricionistas), apresentou 67 respostas positivas referentes à avaliação de sua qualidade de vida como boa e 7 respostas para muito boa ${ }^{(14)}$.

Na Tabela 2 evidencia-se que mais da metade dos profissionais pesquisados não trabalham no SAMU com exclusividade. A partir desse resultado, subentende-se que quase $60 \%$ deles atuam em outros locais, favorecendo a ocorrência do estresse e dos efeitos dele decorrentes, secundário à sobrecarga de trabalho, podendo repercutir na qualidade de vida desses profissionais.

Tabela 2 - Dedicação e opção dos profissionais ao SAMU, segundo avaliação da satisfação com sua saúde. Florianópolis, Santa Catarina. Out-2008

\begin{tabular}{llccccc}
\hline Avaliação da saúde & & Muito ruim & $\begin{array}{c}\text { Nem ruim, } \\
\text { nem boa }\end{array}$ & Boa & Muito boa & Total \\
\cline { 3 - 7 } & & $\mathrm{n}(\%)$ & $\mathrm{n}(\%)$ & $\mathrm{n}(\%)$ & $\mathrm{n}(\%)$ & $\mathrm{n}(\%)$ \\
\hline Dedicação & $\begin{array}{l}\text { Com } \\
\text { exclusividade }\end{array}$ & $1(2,4)$ & $1(2,4)$ & $14(31,1)$ & $1(2,4)$ & $17(41,5)$ \\
& $\begin{array}{l}\text { Sem } \\
\text { exclusividade }\end{array}$ & $1(2,4)$ & $6(14,6)$ & $13(31,7)$ & $4(9,8)$ & $24(58,5)$ \\
Opção & Sim & $2(4,9)$ & $6(14,6)$ & $21(51,2)$ & $5(12,2)$ & $34(82,9)$ \\
& Não & - & $1(2,4)$ & $6(14,6)$ & - & $7(17,1)$ \\
\hline
\end{tabular}

Ao realizar o cruzamento das variáveis dedicação dos profissionais ao SAMU segundo avaliação referente à satisfação com sua saúde, observa-se que dos 58,5\% profissionais que atuam sem exclusividade, 32\% avaliam sua saúde como boa e 17\% como muito ruim ou nem ruim, nem boa. A referida Tabela 2 também apresenta a opção dos profissionais em atuar no referido serviço. Verifica-se que a maioria deles optou por atuar no SAMU e esse resultado é positivo, demonstrando que os profissionais gostam de atuar no referido local, o que pode contribuir no sentido de melhor enfrentamento das dificuldades vivenciadas no ambiente de trabalho.

Quando observadas conjuntamente as variáveis opção por atuar no SAMU e a avaliação dos pesquisados em relação à satisfação com sua saúde, observa-se que dos 82,9\% que optaram por atuar no referido local, 51\% avaliam como boa sua satisfação com a saúde e $20 \%$ como muito ruim ou nem ruim, nem boa. 
Tabela 3 - Dedicação e opção dos profissionais ao SAMU, segundo avaliação da satisfação com sua qualidade de vida. Florianópolis, Santa Catarina. Out-2008

\begin{tabular}{llcccccc}
\hline $\begin{array}{l}\text { Avaliação da qualidade } \\
\text { de vida }\end{array}$ & $\begin{array}{c}\text { Muito } \\
\text { insatisfeito }\end{array}$ & Insatisfeito & $\begin{array}{c}\text { Nem satisfeito, } \\
\text { nem insatisfeito }\end{array}$ & Satisfeito & $\begin{array}{c}\text { Muito } \\
\text { satisfeito }\end{array}$ & Total \\
\cline { 2 - 7 } & $\mathrm{n}(\%)$ & $\mathrm{n}(\%)$ & $\mathrm{n}(\%)$ & $\mathrm{n}(\%)$ & $\mathrm{n}(\%)$ & $\mathrm{n}(\%)$ \\
\hline Dedicação & $\begin{array}{l}\text { Com } \\
\text { exclusividade }\end{array}$ & - & - & $2(4,9)$ & $11(26,8)$ & $4(9,8)$ & $17(41,5)$ \\
& Sem & & & & & & \\
& exclusividade & $2(4,9)$ & $3(7,3)$ & $2(4,9)$ & $16(39,0)$ & $1(2,4)$ & $24(58,5)$ \\
Opçãa & Sim & $2(4,9)$ & $3(7,3)$ & $3(7,3)$ & $21(51,2)$ & $5(12,2)$ & $34(82,9)$ \\
& Não & - & - & $1(2,4)$ & $6(14,6)$ & - & $7(17,1)$ \\
\hline
\end{tabular}

Na Tabela 3 é apresentado o resultado do cruzamento das variáveis Dedicação e Opção dos profissionais ao SAMU, segundo avaliação da satisfação com sua qualidade de vida. Observa-se que dos $58,5 \%$ que se dedicam sem exclusividade, 39\% deles está satisfeito com sua qualidade de vida e $17 \%$ insatisfeito ou indiferente. Dos 82,9\% que optaram por atuar no referido local, 63,4\% sentem-se satisfeitos com sua qualidade de vida e $20 \%$ insatisfeitos ou indiferentes. Verifica-se que mais da metade dos que optaram por atuar no referido serviço, estão satisfeitos com seu trabalho e avaliam sua qualidade de vida como boa.

Na sequência, são apresentadas as Tabelas 4 e 5, resultantes do cruzamento das seguintes variáveis: QV conforme a idade e gênero e avaliação da saúde conforme idade e gênero.

Tabela 4 - Avaliação da Qualidade de Vida conforme a idade e o gênero dos profissionais do SAMU. Florianópolis, Santa Catarina. Out-2008

\begin{tabular}{lccccccc}
\hline Avaliação & \multicolumn{3}{c}{ Idade em anos } & \multicolumn{3}{c}{ Gênero } \\
\cline { 2 - 8 } & $25+-30$ & $30+-35$ & $35+-40$ & $40+-45$ & 45 ou mais & Feminino & Masculino \\
& $\mathrm{n}(\%)$ & $\mathrm{n}(\%)$ & $\mathrm{n}(\%)$ & $\mathrm{n}(\%)$ & $\mathrm{n}(\%)$ & $\mathrm{n}(\%)$ & $\mathrm{n}(\%)$ \\
\hline Ruim & - & $1(2,4)$ & $1(2,4)$ & - & - & - & $2(4,9)$ \\
Nem ruim, nem & $1(2,4)$ & $5(12,2)$ & - & $1(2,4)$ & - & $3(7,3)$ & $4(9,8)$ \\
boa & $10(24,4)$ & $7(17,1)$ & $4(9,8)$ & $4(9,8)$ & $2(4,9)$ & $15(36,6)$ & $12(29,3)$ \\
Boa & - & $1(2,4)$ & $3(7,3)$ & $1(24)$ & - & $2(4,9)$ & $3(7,3)$ \\
Muito boa & $11(26,8)$ & $14(34,1)$ & $8(19,5)$ & $6(14,6)$ & $2(4,9)$ & $20(48,8)$ & $21(51,2)$ \\
\hline Total & & & & & & &
\end{tabular}

Na Tabela 4 é explicitada a avaliação da QV dos profissionais conforme a idade e gênero. Nesta constata-se que dos sujeitos que estão na faixa etária de 25 a 35 anos incompletos, a maioria avalia como nem boa nem ruim ou como boa e nas demais faixas etárias a concentração está na avaliação da mesma como boa.

Ainda em relação aos dados contidos na tabela 4, quanto à avaliação da QV conforme o gênero, evidencia-se que somente os homens (percentual pequeno) avaliam a QV como ruim. A avaliação da QV dos dois gêneros, como nem boa nem ruim, é aproximada em ambos. Importante ressaltar que a avaliação da QV como boa é que teve maior percentual em ambos os gêneros, demonstrando que este grupo de profissionais está satisfeito com sua QV.

Na Tabela 5 é apresentada a avaliação da saúde sob a ótica dos profissionais, também conforme a idade e gênero. Independentemente da idade dos profissionais, o percentual maior em cada uma das faixas etárias se concentrou na avaliação satisfeito com a saúde. No cruzamento das variáveis avaliação da saúde conforme o gênero, observou-se que os maiores percentuais referem-se a satisfação com a saúde, sendo 34,1\% referente ao gênero masculino e $31,7 \%$ ao feminino, perfazendo um total de $65,8 \%$, considerando ambos os gêneros dos participantes da pesquisa.

Importante destacar que a avaliação que o 
profissional faz de sua saúde bem como de sua QV podem interferir na percepção dos estressores no ambiente de trabalho e, inclusive, nos mecanismos de enfrentamento utilizados para lidar com os mesmos.

Tabela 5 - Avaliação da saúde conforme a idade e o gênero dos profissionais do SAMU. Florianópolis, Santa Catarina. Out-2008

\begin{tabular}{|c|c|c|c|c|c|c|c|}
\hline \multirow[t]{2}{*}{ Avaliação } & \multicolumn{5}{|c|}{ Idade em anos } & \multicolumn{2}{|c|}{ Gênero } \\
\hline & $\begin{array}{c}25 \text { |-- } 30 \\
\mathrm{n}(\%) \\
\end{array}$ & $\begin{array}{c}30 \mid--35 \\
\mathrm{n}(\%) \\
\end{array}$ & $\begin{array}{c}35 \text { |-- } 40 \\
\mathrm{n}(\%) \\
\end{array}$ & $\begin{array}{c}40 \mid--45 \\
\mathrm{n}(\%) \\
\end{array}$ & $\begin{array}{c}45 \text { ou mais } \\
\mathrm{n}(\%)\end{array}$ & $\begin{array}{c}\text { Feminino } \\
\mathrm{n}(\%)\end{array}$ & $\begin{array}{c}\text { Masculino } \\
\mathrm{n}(\%)\end{array}$ \\
\hline Muito insatisfeito & $1(2,4)$ & $1(2,4)$ & - & - & - & $1(2,4)$ & $1(2,4)$ \\
\hline Insatisfeito & - & $2(4,9)$ & - & $1(2,4)$ & - & $2(4,9)$ & $1(2,4)$ \\
\hline $\begin{array}{l}\text { Nem satisfeito, } \\
\text { nem insatisfeito }\end{array}$ & $2(4,9)$ & $2(4,9)$ & - & - & - & $1(2,4)$ & $3(7,3)$ \\
\hline Satisfeito & $7(17,1)$ & $7(17,1)$ & $6(14,6)$ & $5(12,2)$ & $2(4,9)$ & $14(34,1)$ & $13(31,7)$ \\
\hline Muito satisfeito & $1(2,4)$ & $2(4,9)$ & $2(4,9)$ & - & - & $2(4,9)$ & $3(7,3)$ \\
\hline Total & $11(26,8)$ & $14(34,1)$ & $8(19,5)$ & $6(14,6)$ & $2(4,9)$ & $20(48,8)$ & $21(51,2)$ \\
\hline
\end{tabular}

Uma investigação das relações entre trabalho, saúde e condições de vida de 10 profissionais de enfermagem (enfermeiras, técnicas e auxiliares), mostrou que todas referiram ter realizado algum tratamento médico por depressão e nervosismo ${ }^{(15)}$. Nesse contexto, os fatores que influenciam a QV podem estar relacionados à atividade laboral do individuo, mas também às condições de trabalho oferecidas pela organização em que está inserido ${ }^{(16)}$. Contribuindo, a falta de tempo para lazer e atividades físicas pode prejudicar a QV, pois estas ajudam a manter a saúde, tanto física quanto mental dos trabalhadores ${ }^{(17)}$.

\section{CONSIDERAÇÕES FINAIS}

O perfil da equipe pesquisada é: homens e mulheres, em percentuais aproximados, jovens, casados, com filhos, especialistas, enfermeiros e médicos. Eles possuem de 5 a 10 de profissão, a maioria optou por atuar no SAMU, onde está há menos de 5 anos e, mais da metade, sem exclusividade. Sentemse satisfeitos com sua saúde e avaliam sua qualidade de vida de forma positiva.

Ao analisar conjuntamente a variável avaliação dos pesquisados em relação à satisfação com sua saúde segundo a dedicação e opção pelo SAMU, observase que dos trabalhadores que atuam sem exclusividade, a avaliação da maioria deles em relação a sua saúde é boa, mas é preocupante o percentual de $17 \%$ que a avalia como muito ruim ou nem ruim, nem boa. Esta análise apresenta percentuais semelhantes quando analisada segundo a opção em trabalhar no SAMU.

Observa-se, de forma geral, que os pesquisados avaliam sua qualidade de vida como boa e sentem-se satisfeitos com sua saúde, independente da idade e do gênero.

Conhecer a avaliação sobre a saúde e qualidade de vida, segundo o perfil dos trabalhadores de um Serviço de Atendimento Pré-Hospitalar é importante, possibilita reflexões e ações, tanto de gestores que atuam em APH, quanto dos demais profissionais, visando preservar a saúde e qualificar a assistência aos usuários que acessam o referido serviço. Os resultados dessa pesquisa podem ser igualmente importantes no sentido de instigar e estimular pesquisadores e estudantes a realizar mais investigações envolvendo a referida temática.

\section{REFERÊNCIAS}

1. Ladeira RM, Barreto SM. Fatores associados ao uso de serviço de atenção pré-hospitalar por vítimas de acidentes de trânsito. Cad Saúde Publ. 2008;24(2):28794.

2. Pereira WAP, Lima MADS. Atendimento pré-hospitalar: caracterização das ocorrências de acidente de trânsito. Acta Paul Enferm. 2006;9(3):279-83.

3. Lentz RA, Costenaro RGS, Gonçalves LHT, Nassar SN. O Profissional de enfermagem e a qualidade de vida: uma abordagem fundamentada nas dimensões propostas por Flanagem. Rev Latino-Am Enferm. 2000;8(4):7-14. 
4. Dejours C. A loucura do trabalho: estudo sobre a psicopatologia do trabalho. São Paulo: Cortez; 1992

5. Ministério da Saúde (BR). Conselho Nacional da Saúde. Diretrizes e normas regulamentadoras da pesquisa envolvendo os seres humanos: Resolução 196/96. Brasília, 1996.

6. Rossi AM. Estressado, eu? Porto Alegre: RBS Publicações; 2004.

7. Carlotto MS, Nakamura AP, Câmara SG. Síndrome de Burnout em estudantes universitários da área da saúde. Psicol. 2006;37(1):57-62.

8. Guido LA. Stress e coping entre enfermeiros de centro cirúrgico e recuperação anestésica [tese]. São Paulo (SP): Escola de Enfermagem da Universidade de São Paulo; 2003.

9. Rebouças D, Legay LT, Abelha L. Satisfação com o trabalho e impacto causado nos profissionais de Serviço de Saúde Mental. Rev Saúde Publ. 2007;41(2):244-50

10. Resende E, Takershina ML. RH em tempo real: conceitos e ferramentas modernas para gestão de recursos humanos. Rio de Janeiro: Quality Mark; 2000.

11. Meira MM. Diretrizes para a educação permanente no Serviço de Atendimento Móvel de Urgência (SAMU) [dissertação]. Florianópolis (SC): Universidade Federal de Santa Catarina. Pós-Graduação em Enfermagem; 2007.

12. Martinez MC, Paraguay AIBB, Latorre MRDO. Relação entre satisfação com aspectos psicossociais e saúde dos trabalhadores. Rev Saúde Publ. 2004;38(1):55-61.

13. Paschoa S, Zanei SSV, Whitaker IY. Qualidade de vida dos trabalhadores de enfermagem de unidades de terapia intensiva. Acta Paul Enferm. 2007;20(3):305-10.

14. Spiller APM, Dyniewicz AM, Slomp MGFS. Qualidade de vida de profissionais da saúde em hospital universitário. Cogitare Enferm. 2008;13(1):88.

15. Elias MA, Navarro VL. A relação entre o trabalho, a saúde e as condições de vida: negatividade e positividade no trabalho das profissionais de enfermagem de um hospital escola. Rev Latino-Am Enferm. 2006;14(4):517-25.

16. Pizzoli LML. Qualidade de vida no trabalho: um estudo de caso de enfermeiras do Hospital Heliópolis. Ciênc Saúde Col. 2005;10(4):1055-62.

17. Schmidt DRC, Dantas RAS. Qualidade de vida no trabalho de profissionais de enfermagem, atuantes em unidades do bloco cirúrgico, sob a ótica da satisfação. Rev Latino-Am Enferm. 2006;14(1):54-60. 